\title{
Radar Signal Processing Method Based on the Lateral Distance Element Synthesis Processing
}

\author{
Rongrong Zhang ${ }^{1}$ \\ 1.Department of Mechanical and Electrical Engineering, Jiangsu Polytechnic of Finance and \\ Economics, Jiangsu Huai'an, 223001, China
}

\begin{abstract}
Keywords: Ultra Wideband; Spatial resolution, Compressive sampling; Peak signal-to-noise ratio; Temporal correlation; Imaging Technology, Radar Signal
\end{abstract}

\begin{abstract}
Ultra Wideband (UWB: Ultra-Wideband) with high spatial resolution and positioning measurement, has the ability of suppressing the multi-path effect and penetration loss medium. It contains abundant target information in the received signal. The compressed sensing theory is introduced into the imaging technology of radar signal. In order to reduce the number of the traditional algorithm of tracking compressive sampling matching, improve measurement precision reconstruction, a compressed sensing based on peak signal-to-noise ratio compressive sampling matching tracking algorithm is proposed. Based on a ultra wideband detection technology, and using the temporal correlation between the data to using compress, we propose an local adaptive estimate threshold. Finally, comparison and analysis with the conventional image processing effect, it shows that the algorithm improves people accuracy of ultra wideband radar imaging judgment. It promotes the development and application of ultra wideband radar also.
\end{abstract}

\section{Introduction}

Ultrasonic imaging and logging technology is widely applied in engineering field. At present, the newest logging technology in the world is the three-dimensional signature logging method firstly developed and researched in U.S. Specifically, the underground array sensors are used to scan along the longitudinal and radial directions of the well to acquire lots of stratum information and then image processing method is used to obtain the three-dimensional image around the well or the two-dimensional image [1] of the well wall. Therefore, the target image obtained through the method is more visual, accurate and convenient than that obtained through curve mode. However, the ultrasonic imaging and logging equipment usually has sudden stoppage during the movement thereof for imaging and logging due to the disturbance of uneven underground resistance and the logging equipment will repeatedly scan at the sudden stoppage depth for imaging due to the existence of the elastic deformation of the logging cable, yet the corresponding depth measuring instrument is still in constant motion to cause the unmatched logging data and depth position, namely the common strap chain area, and such phenomenon is called "chain effect" [2]. In order to solve the problem, Bertalmio et al. [3] have adopted partial differential equation to repair the image obtained through ultrasonic logging and imaging, and Chan, et al. [4] have adopted total variation(TV) model to repair the image obtained through ultrasonic logging and imaging. Meanwhile, Criminisi, et al. [5] have adopted imaging texture to design logging image repairing algorithm, and many scholars have subsequently proposed a lot of improvement methods and obtained certain effect. Generally, these repairing schemes have good repairing effect for curve or small image area, but have dissatisfactory repairing effect for the image with large abnormal area. 


\section{Wavelet Domain Image Repairing Algorithm}

As mentioned above, the sudden stoppage of ultrasonic logging and imaging is mainly resulted from the uneven underground resistance and accordingly leads to the unmatched logging data and depth position. The common engineering method is obtained according to the interpolation of the measurement data of the upper and lower edges of the chain area and practice has proven that such interpolation method has good repairing effect for processing narrow chains but dissatisfactory repairing effect for large chain area. The image is assumed to be expressed as:

$$
u(x)=u_{0}(x)+n(x)
$$

In the formula, ${ }^{u_{0}(x)}$ is ultrasonic logging original image, ${ }^{n(x)}$ is image noise which usually refers to Gaussian noise in experimental environment. If the image size is assumed as $n \times m$, then the standard orthogonal wavelet transformation form is:

$$
u(\beta, x)=\sum_{j, k} \beta_{i, k} \phi_{i, k}(x), j \in Z, k \in Z^{2}
$$

In wavelet transformation image expression, $\beta=\left\{\beta_{j, k}\right\}$ is wavelet coefficient. Notably, high and low frequencies are not distinguished for the coefficient, and there are two reasons for such processing mode: the first one is to simplify calculation, and the second one is to allow certain coefficient loss within high and low frequency ranges. In practical application, noise and data packet losses are unavoidable and accordingly cause wavelet coefficient loss, and meanwhile the remaining coefficient is also influenced by noise. In order to accurately express wavelet domain image, the noisy total variation wavelet domain image repairing algorithm model proposed in Literature is:

$$
\min F\left(u, u_{0}\right)=\int|\nabla u(\beta, x)| d x+\sum \lambda_{i, k}\left(\beta_{j, k}-\alpha_{j, k}\right)^{2}
$$

In the formula, when there is no noise, then $\lambda=0$; if $j, k \in D$ and $D$ belongs to the image area to be repaired, then ${ }^{\lambda_{j, k}}=0$; if $j, k \notin D$, then ${ }^{\lambda_{j, k}}$ is positive constant. Introduce time variable ${ }^{t}$ and adopt gradient descent method to obtain wavelet coefficient:

$$
\left(\beta_{i, k}\right)_{t}=\int \nabla\left(\frac{\nabla u}{|\nabla u|}\right) \varphi_{i, k}(x) d x-2 \lambda_{i, k}\left(\beta_{i, k}-\alpha_{i, k}\right)
$$

In the formula, $\int \nabla(\nabla u /|\nabla u|) \varphi_{i, k}(x) d x$ stands for the projection of the image curvature in wavelet domain. According to the analysis of total variation wavelet domain image repairing model, the total variation characteristics and the calculation reliability can significantly influence the image repairing effect during the conversion process of wavelet domain and image pixels, as shown in Fig. 1.

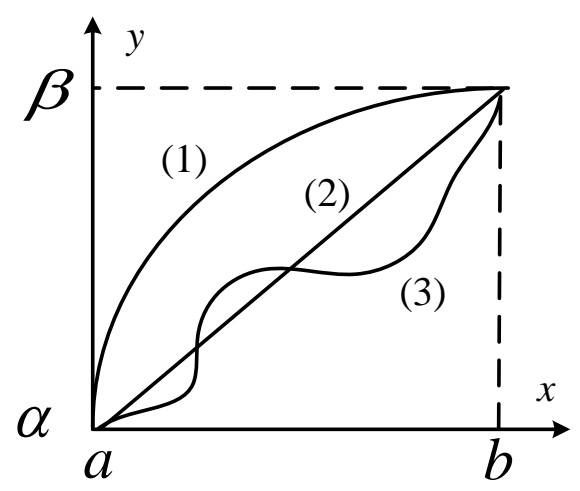

Fig. 1. Total Variation Characteristics

Fig. 1 shows three monotone increasing curvilinear (linear) functions. As shown in Fig. 1, if $u \in[a, b]$ and the functions are monotone increasing functions in the range of ${ }^{[a, b]}$ and can meet the conditions $u(a)=\alpha$ and $u(b)=\beta$, then the above three functions have the same total variation:

$$
\int\left|\nabla u_{1}\right| d x=\int\left|\nabla u_{2}\right| d x=\int\left|\nabla u_{3}\right| d x
$$


But the three segments are morphologically different from each other, and especially when modelis used to repair, the calculations of wavelet coefficient and curvature are not in the same domain, so the adoption of total variation method for image repairing may cause "piecewise constant effect" in the steady-state value of the wavelet coefficient and influence the image repairing effect.

\section{Experimental Results and Analysis}

The simulation data are from AER 7\# ultrasonic logging image, and AER 7\# well is located in Erlian Basin, the NeiMonggol Autonomous Region and belongs to North China Oil Field Erlian Company at XiIinGoI Prairie. Fig. 2 includes original image data, "chain area" automatic positioning and identification simulation image and the comparison of the images repaired by three wavelet algorithms. Simulation platform: CPU: AMD AthlonII X4 760K, memory: 4G, and operating system: win 7.

$d b 7 / 9$ biorthogonal wavelet is adopted as the simulation parameter, the image noise parameter is $\lambda=0.05$ [10 11], and the results of the original ultrasonic logging image repaired by algorithms are shown in Fig. 2.

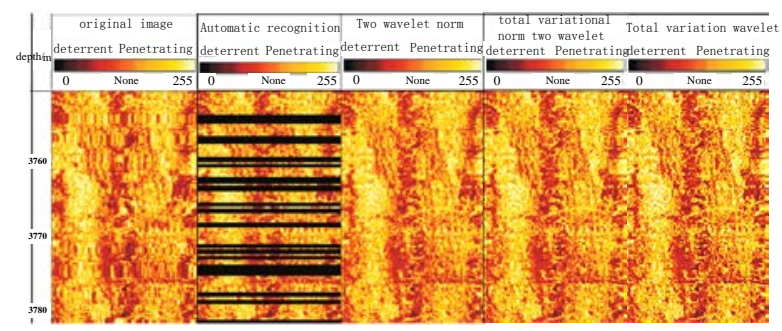

Fig. 2. Automatic Identification and Effect Comparison of Three Wavelet Algorithms

As shown in Fig. 2, the leftmost original logging image has obvious abnormal chain area and such phenomenon widely exists in actual ultrasonic logging images; the second picture presents the chain identification area provided through the chain area automatic identification algorithms, the black lines stand for the chain areas which are accurately identified as shown in the picture. As previously mentioned, the total variation wavelet focuses on edge preservation but 2-norm wavelet tends to the smooth image repairing, so the experiment aims at showing the comparison results of the three wavelet processing algorithms. According to the right three pictures shown in Fig. 2, 2-norm wavelet has smooth repairing effect but has edge burrs among textures; the total variation wavelet focuses on edge preservation, so the image repaired thereby has clearer texture; the total variation 2-norm wavelet has intermediate repairing results and well balances the requirements for image repairing smoothness and edge preservation. In conclusion, the total variation 2-norm wavelet has improved image repairing effect on the basis of original wavelet characteristics.

Fig. 3 visually shows the result comparison of original ultrasonic logging image repaired by three algorithms. From the aspect of objective data, the following paragraph will compare the change relation between the wavelet coefficient loss rate[13] and PSNR (peak signal-to-noise ratio) parameters of the total variation 2-norm wavelet image repairing method, the total variation wavelet image repairing method and interpolation image repairing method[12] used for repairing ultrasonic logging image, as shown in Fig. 2.

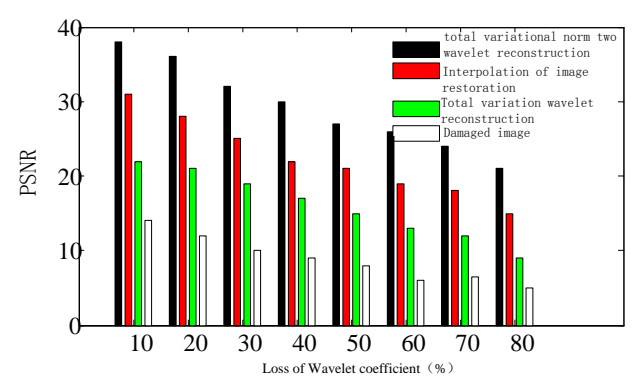

Fig. 3. PSNR Comparison Result 
According to Fig. 4, PSNR parameter of the ultrasonic logging image repaired by the total variation 2-norm wavelet image repairing algorithm is better than that repaired by interpolation image repairing algorithm, and the two are better than the total variation wavelet image repairing algorithm and meanwhile PSNR of the damaged image is lowest. Therefore, it is indicated that the three algorithms have certain effect for image repairing, but the total variation 2-norm wavelet algorithm is obviously better than the other two algorithms. Meanwhile, along with the increase of wavelet coefficient loss rate, PSNRs of the images repaired by these algorithms are all reduced and accordingly indicate the severe damage of the images, wherein the more severely the image is damaged, the smaller the PSNR is.

In the aspect of algorithm operating efficiency, Fig. 3 shows the change relation between the wavelet coefficient loss rate and the operating time of the three algorithms under the same conditions, namely the total variation 2-norm wavelet image repairing algorithm, the total variation wavelet image repairing algorithm and the interpolation image repairing algorithm. In order to stabilize the simulation results, the average operating time of each algorithm for 30 operations is shown in the following figure.

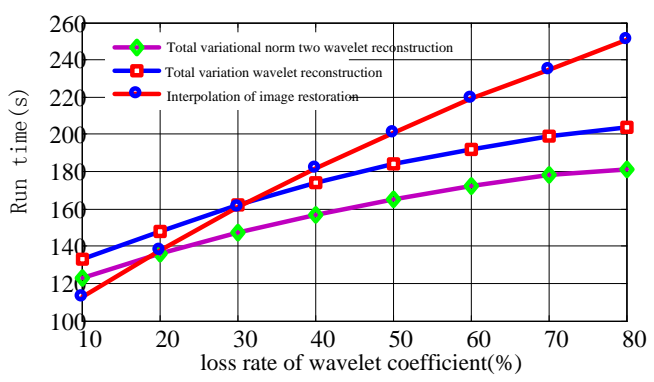

Fig. 4. Algorithm Operating Time Comparison Result

As shown in Fig. 4, the operating time of the three algorithms is prolonged along with the increase of wavelet coefficient loss rate and accordingly indicates that the image is more severely damaged, and this is also consistent with the objective reality. When the wavelet coefficient loss rate is low, the interpolation image repairing algorithm has fast operating speed and the operating time thereof is even shorter than that of the wavelet algorithm; along with the increase of the loss rate, the operating time of the interpolation image repairing algorithm is significantly prolonged to exceed that of the total variation 2-norm wavelet image repairing algorithm and the total variation wavelet image repairing algorithm, and the operating time of the latter two is basically the same, but the total variation 2-norm wavelet image repairing algorithm is a little better than the total variation wavelet image repairing algorithm, because the improved algorithm mode has enhanced the repairing efficiency.

\section{Conclusion}

In allusion to the problem that the traditional image repairing algorithm is not ideally effective for the abnormal strap area in ultrasonic imaging and logging application, the improved traditional total variation wavelet image repairing algorithm is introduced to solve the repairing problem of ultrasonic logging image with relatively large abnormal strap area. The experimental result shows that the improved algorithm is obviously better than the comparison algorithms in the aspects of repairing effect, operating efficiency, etc. In future, the research will be focused on the wavelet repairing algorithm. Known from Fig. 2, the repairing effect of the algorithm still can be further improved, so the repairing effect improvement is the research emphasis in future. 


\section{References}

[1] Zhang, Mengxin, Zhihan Lv, Xiaolei Zhang, Ge Chen, and Ke Zhang. "Research and Application of the 3D Virtual Community Based on WEBVR and RIA." Computer and Information Science 2, no. 1 (2009): p84.

[2] Su, Tianyun, Zhihan Lv, Shan Gao, Xiaolong Li, and Haibin Lv. "3D seabed: 3D modeling and visualization platform for the seabed.” In Multimedia and Expo Workshops (ICMEW), 2014 IEEE International Conference on, pp. 1-6. IEEE, 2014.

[3] Li, Xiaoming, Zhihan Lv, Baoyun Zhang, Weixi Wang, Shengzhong Feng, Jinxing Hu. "WebVRGIS Based City Bigdata 3D Visualization and Analysis." In Pacific Visualization Symposium (PacificVis), 2015 IEEE. IEEE, 2015.

[4] S. Li, Y. Geng, J. He, K. Pahlavan, “Analysis of Three-dimensional Maximum Likelihood Algorithm for Capsule Endoscopy Localization,” 2012 5th International Conference on Biomedical Engineering and Informatics (BMEI), Chongqing, China Oct. 2012 (page 721-725).

[5] Y. Geng, J. He, H. Deng and K. Pahlavan, "Modeling the Effect of Human Body on TOA Ranging for Indoor Human Tracking with Wrist Mounted Sensor," 16th International Symposium on Wireless Personal Multimedia Communications (WPMC), Atlantic City, NJ, Jun. 2013. 